\section{Efectividad de las intervenciones para revertir el diagnóstico del síndrome metabólico: actualización de un metaanálisis de comparación mixta de tratamientos}

Adriana Guzmán'1, Esteban Navarro1, Leidy Obando1', Jorge Pacheco', Korina Quirós', Leticia Vásquez ${ }^{1}$, Milena Castro',2, Fernando Ramírez ${ }^{1}$

${ }^{1}$ Escuela de Estadística, Facultad de Ciencias Económicas, Universidad de Costa Rica, San José, Costa Rica

${ }^{2}$ Centro de Investigación en Matemática Pura y Aplicada, Universidad de Costa Rica, San José, Costa Rica

Introducción. El conocer las intervenciones más efectivas para revertir el síndrome metabólico es clave para el diseño de estrategias clínicas de prevención de enfermedades como la diabetes mellitus de tipo 2 y la enfermedad cardiovascular.

Objetivo. Sintetizar el tamaño del efecto de las intervenciones disponibles para revertir un diagnóstico de síndrome metabólico.

Materiales y métodos. Se hizo la búsqueda en Embase y Medline, incluyendo los ensayos clínicos en los que la variable "respuesta" se definía como la reversión del diagnóstico del síndrome metabólico. Se categorizaron las intervenciones en cuatro dimensiones: 1) estilo de vida (dieta y ejercicio); 2) farmacia; 3) combinación de estilo de vida y farmacia, y 4) grupos de control; finalmente, se hizo una comparación mixta de tratamientos.

Resultados. Se detectaron dos estudios adicionales a los incluidos en el metaanálisis publicado por Dunkley, et al., en el 2012. Se estimó que las intervenciones relacionadas con el estilo de vida tuvieron 2,61 veces (intervalo de credibilidad entre 1,00 y 5,47) más probabilidades de revertir el síndrome metabólico que las de los grupos de control y las relacionadas con los tratamientos farmacéuticos, una probabilidad de 3,39 veces más que las del grupo de control, pero con un intervalo de credibilidad entre 0,81 y 9,99. Las intervenciones sobre el estilo de vida tuvieron 1,59 veces más probabilidades de revertir el síndrome metabólico que las del tratamiento farmacéutico.

Conclusión. Las estrategias basadas en la dieta y la actividad física de las personas, tuvieron una mayor probabilidad de ser más efectivas para revertir el diagnóstico de síndrome metabólico.

\section{Citación:}

Guzmán A, Navarro E, Obando L, Pacheco J, Quirós $\mathrm{K}$, Vásquez L, et al. Efectividad de las intervenciones para revertir el diagnóstico del síndrome metabólico: actualización de un metaanálisis de comparación mixta de tratamientos. Biomédica. 2019;39:647-62. https://doi.org/10.7705/biomedica.4684

\section{Correspondencia:}

Milena Castro, Escuela de Estadística, Facultad de Ciencias Económicas, Universidad de Costa Rica, Sede Rodrigo Facio, San Pedro, Montes de Oca,

San José, Costa Rica

Teléfono: (506) 25116528

milena.castromora@ucr.ac.cr

\section{Contribución de los autores:}

Milena Castro y Fernando Ramírez: diseño del análisis

Adriana Guzmán, Esteban Navarro, Leidy Obando, Jorge Pacheco, Korina Quirós y Leticia Vásquez: selección y extracción de los estudios

Todos los autores participaron en el análisis de los resultados y en la escritura del manuscrito.

Financiación:

No se invirtió ningún tipo de fondos para llevar a cabo el estudio.

\section{Conflicto de intereses:}

Los autores declaran que no tienen ningún conflicto de interés.
Palabras clave: síndrome metabólico; diabetes mellitus de tipo 2; enfermedades cardiovasculares; metaanálisis; oportunidad relativa.

Effectiveness of interventions for the reversal of a metabolic syndrome diagnosis:

Introduction: Identifying the most effective interventions to reverse the metabolic syndrome can be key in the design of clinical strategies to prevent progression to type 2 diabetes mellitus and cardiovascular disease.

Objective: To estimate the effect size of the interventions used for the reversal of metabolic syndrome.

Materials and methods: We searched in Embase and Medline databases for randomized clinical trials with an outcome defined as the reversal of the metabolic syndrome diagnosis. We classified the interventions in four dimensions: 1) lifestyle (diet and exercise); 2) pharmaceuticals; 3) a combination of both, and 4) control groups, and we conducted a mixed treatment comparison analysis.

Results: Additional to the previous meta-analysis published by Dunkley, et al. in 2012, we identified two other studies. Lifestyle interventions had 2.61 more chances to achieve the reversal of the metabolic syndrome than the control group, with a credible interval between 1.00 and 5.47. Pharmaceutical treatments showed a 3.39 higher chance of reversing the syndrome compared with the control group, but the credible interval was estimated from 0.81 to 9.99 . Lifestyle interventions had 1.59 more chance of reversal than the pharmaceutical treatments.

Conclusion: Diet and physical activity-based interventions had a higher probability of effectiveness to reverse a metabolic syndrome diagnosis.

Keywords: Metabolic syndrome; diabetes mellitus, type 2; cardiovascular diseases; metaanalysis; odds ratio. An update of a meta-analysis of mixed treatment comparison studies 
Los estudios sobre el síndrome metabólico adoptan las definiciones planteadas por la Organización Mundial de la Salud (OMS), la International Diabetes Federation (IDF) y el National Cholesterol Education Program Adult Treatment Panel III (NCEP-ATP III) (NCEP). Según este último, el síndrome metabólico se define como la agrupación, por lo menos, de tres factores de riesgo cardiovascular de una lista de cinco (glucosa elevada en plasma en ayuno, presión arterial elevada, triglicéridos elevados, niveles bajos de HDL y obesidad abdominal según los valores de la circunferencia de la cintura), en tanto que la elevación de la insulina constituye un factor relevante en la definición de la OMS y la obesidad abdominal es el factor principal para la IDF (1).

Las estimaciones de la prevalencia del síndrome metabólico se ven influenciadas directamente por el tipo de definición utilizada y las variaciones que se presentan al medir la obesidad; por ejemplo, en Francia se reporta una prevalencia de $10 \%$ en hombres y de $7 \%$ en mujeres con edades entre los 30 y los 64 años al emplear los parámetros del NCEP y, de 23,5\%, aproximadamente, en hombres y alrededor del 9,2\% en mujeres en el mismo rango de edad, al emplear los de la OMS (2).

La divergencia según las definiciones introduce una considerable heterogeneidad en la comparación de la prevalencia a nivel mundial (3). Sin embargo, con la definición del NCEP hay estudios en países como México, con el 26,6 \% para hombres y mujeres entre los 20 y los 69 años de edad (4); en Irlanda, se ha estimado una prevalencia del síndrome metabólico de 21,8 $\%$ para hombres y de $21,5 \%$ para mujeres entre los 50 y los 69 años de edad (5); en Estados Unidos, se ha estimado una prevalencia de $24,2 \%$ en hombres y de $23,5 \%$ en personas mayores de 19 años (6), y en India, de 7,9\% en hombres y de 17,5\% en mujeres mayores de 20 años (7), entre otros (3).

La investigación sobre el síndrome metabólico se ha tornado crucial para la prevención de la diabetes de tipo 2 (8), por lo que el uso de una definición que no considere los altos niveles de insulina es crucial en el análisis detallado del fenómeno y para proponer estrategias de salud pública. Asimismo, este síndrome está asociado con un riesgo moderado de desarrollar enfermedades cardiovasculares $(9,10)$ y con un riesgo considerable de desarrollar diabetes de tipo 2 (11); según la actualización de las revisiones llevada a cabo por una de las autoras (en proceso de publicación), se estima que el riesgo relativo de mortalidad por enfermedad cardiovascular en personas con síndrome metabólico es 1,67 veces mayor que en las personas que no lo tienen y, para la diabetes de tipo 2, el riesgo relativo se incrementa hasta 4,31 veces en personas con el síndrome.

En este contexto, el estudio de dicho síndrome resulta de gran relevancia por su relación con la obesidad como factor de riesgo. La detección de los individuos en la práctica clínica es crucial para el planteamiento de políticas públicas de salud a nivel mundial y tiene una gran posibilidad de incidir en la reducción de la prevalencia de la diabetes de tipo 2, los accidentes cerebrovasculares y las enfermedades del corazón en general, tanto a nivel de los incidentes específicos, como de la mortalidad general.

Por lo tanto, el determinar las intervenciones más efectivas es un requisito en los procesos de investigación. En la revisión sistemática de Dunkley, et al. (12), en el 2012, se hizo una primera síntesis de los datos disponibles mediante un metaanálisis de comparaciones mixtas de tratamientos, la cual resulta pertinente, dado que no todas las intervenciones disponibles se han contrastado en forma directa (13). Los tratamientos propuestos para el síndrome metabólico 
se pueden agrupar en estrategias relacionadas con el estilo de vida, con el tratamiento farmacológico o con su combinación. En el estudio de Dunkley, et al., no se establece qué tipo de tratamiento sobresale de forma singular.

En el presente artículo, se presenta una actualización de la evidencia científica que permita especificar mejor la intervención con mayores probabilidades de ser efectiva.

\section{Materiales y métodos}

Se recolectaron los datos secundarios de las publicaciones disponibles cuya pregunta de investigación se hubiera centrado en la observación del tamaño del efecto de las intervenciones y sus diferentes aproximaciones tecnológicas, con el fin de recomendarlas en el marco de las políticas públicas en salud.

Se hizo una revisión sistemática para extraer los datos requeridos y establecer un contraste entre las diferentes opciones tecnológicas, basadas en esquemas de dieta y ejercicio, y de tratamientos farmacológicos (14). Este tipo de datos posibilita el estudio de un parámetro de medición, en este caso, la razón de probabilidades o momios (odds ratio, OR) y la utilización de métodos de síntesis de la evidencia para estimar este tipo de medidas ofrece mayor precisión frente a la heterogeneidad de las poblaciones.

Por lo tanto, se parte de la necesidad de determinar la mejor intervención contra el síndrome metabólico para, así, prevenir el avance acelerado hacia un mayor compromiso de la salud, como la enfermedad cardiovascular o la diabetes mellitus de tipo 2.

La revisión comprendió intervenciones incluidas en alguna de las siguientes cuatro categorías: control, intervenciones sobre el estilo de vida (dieta o ejercicio), tratamientos farmacológicos o la combinación de estas dos últimas. En este sentido, se hizo una búsqueda exhaustiva de la 'evidencia' científica publicada en artículos sobre estudios clínicos cuyos resultados incluyeran la proporción de las personas en cada uno de los grupos de intervención en quienes hubo reversión del síndrome metabólico.

Se siguieron rigurosas estrategias de validación de los datos requeridos en cada etapa del proceso, con el fin de garantizar la calidad de los datos extraídos y analizados.

\section{Estrategia de búsqueda}

En la búsqueda se utilizaron palabras clave en tres categorías: tipo de población representada en la muestra, características de interés relacionadas con el síndrome metabólico y especificaciones metodológicas para la detección de estudios clínicos.

Se definió cada conjunto a partir de la unión de las palabras clave de cada una de las categorías. La población se configuró a partir de las palabras adult, aged, female, male y human. En cuanto a las características de base de cada estudio, se recopilaron los términos metabolic syndrome, metabolic syndrome $X$, glucose, triglycerides, hypertension, obesity, diabetes mellitus, cardiovascular disease, cardiovascular risk y metabolic cardiovascular syndrome. Para recopilar el conjunto de estudios clínicos, se utilizaron los términos article, priority journal, clinical trial, controlled clinical trial, controlled study, randomized controlled trial, randomized trial y placebo. 
Después de definir los conjuntos, se diseñó la estrategia para obtener la intersección de las tres categorías, y los estudios resultantes se clasificaron según los criterios de inclusión y exclusión para obtener aquellos que conformaran la base analítica para determinar la mejor intervención de reversión del síndrome metabólico.

La estrategia de búsqueda se validó con los artículos incluidos en el estudio de base (12), cuyas palabras clave se extrajeron para calcular su frecuencia, con el propósito de incluir solo aquellas que estuvieran presentes, por lo menos, en cinco artículos.

En la segunda etapa, se implementó la estrategia de búsqueda en las bases de datos. Para los documentos en lengua extranjera, se hicieron traducciones parciales o completas para comprobar el cumplimiento de los criterios de inclusión.

La búsqueda se hizo en las bases de datos referenciales de Embase y Medline. La actualización consistió en revisar los estudios relevantes que hubiesen sido publicados entre enero del 2010 y octubre del 2017, ya que el estudio de base había incluido los estudios anteriores a enero del 2010 (12). Una vez se completó la búsqueda en cada base de datos, se excluyeron los duplicados y se diseñó una base de datos para registrar los documentos relevantes para la revisión y el metaanálisis. La búsqueda se validó eliminando el filtro por año y verificando la aparición de los 13 artículos incluidos en el estudio de referencia, los cuales efectivamente se encontraron, con lo cual se validó la estrategia de búsqueda para la actualización.

\section{Selección de los estudios}

La búsqueda arrojó 5.144 títulos en los cuales se usaron los criterios de inclusión y exclusión específicos para seleccionar el tipo de información requerida para comparar las diferentes opciones tecnológicas empleadas en la resolución de una condición con las características del síndrome metabólico. En la figura 1 se presenta el proceso de selección de los estudios.

\section{Criterios de inclusión}

Los criterios de inclusión fueron los siguientes: estudios clínicos controlados en poblaciones humanas mayores de 18 años cuya muestra incluyera una proporción de personas (mayor de 0) con diagnóstico de síndrome metabólico y un seguimiento mayor o igual a 24 semanas. Además, se consideró la inclusión, por lo menos, de dos tipos de intervención de los tres contemplados: el de intervenciones relacionadas con el estilo de vida de las personas (basadas en componentes de dieta, ejercicio o ambas), el de los tratamientos farmacéuticos disponibles, o los que combinaban estilo de vida y farmacoterapia.

Todos los estudios incluidos incorporaban, por lo menos, un nivel de comparación basal o un placebo. No hubo restricción por el idioma y solo se incluyeron aquellos en los que se daba una definición de síndrome metabólico acorde con los criterios especificados por el NCEP.

\section{Criterios de exclusión}

Se revisaron los resúmenes de los artículos para determinar si cumplían con los requisitos del análisis y se excluyeron los que reportaban la medición de un efecto por componente del síndrome, en lugar de un efecto del 
conglomerado de componentes. Se consideraron solo aquellos artículos en los que la definición de la variable 'respuesta' siguiera una distribución binomial, es decir, resultado positivo si la persona del grupo de intervención cumplía con los criterios del diagnóstico del síndrome metabólico, y resultado negativo si la característica de interés estaba ausente.

Se excluyeron todos los artículos que ofrecían una respuesta multivariada, y aquellos que no cumplían con los mismos criterios de inclusión y exclusión definidos por el estudio de referencia (12). Además, se excluyeron los documentos que no ofrecían los datos de extracción especificados para dicho metaanálisis.

\section{Extracción de los datos}

Se diseñó una estrategia para la extracción y la evaluación de la calidad de los datos seleccionados, utilizando las palabras clave de los trece artículos seleccionados en el estudio de base. Se emplearon las funciones condicionales en Excel, para verificar que los artículos seleccionados cumplieran con los términos y los criterios de inclusión y exclusión.

Se consideraron las variables características del estudio y el documento de publicación, el tamaño de muestra, las características demográficas de la población de interés, los tiempos de seguimiento, la proporción relativa de los registros de reversión del síndrome metabólico (porcentaje) o su conteo absoluto. En algunos casos, fue necesario hacer transformaciones para obtener los porcentajes, dado que la medida de interés se reportó como OR.

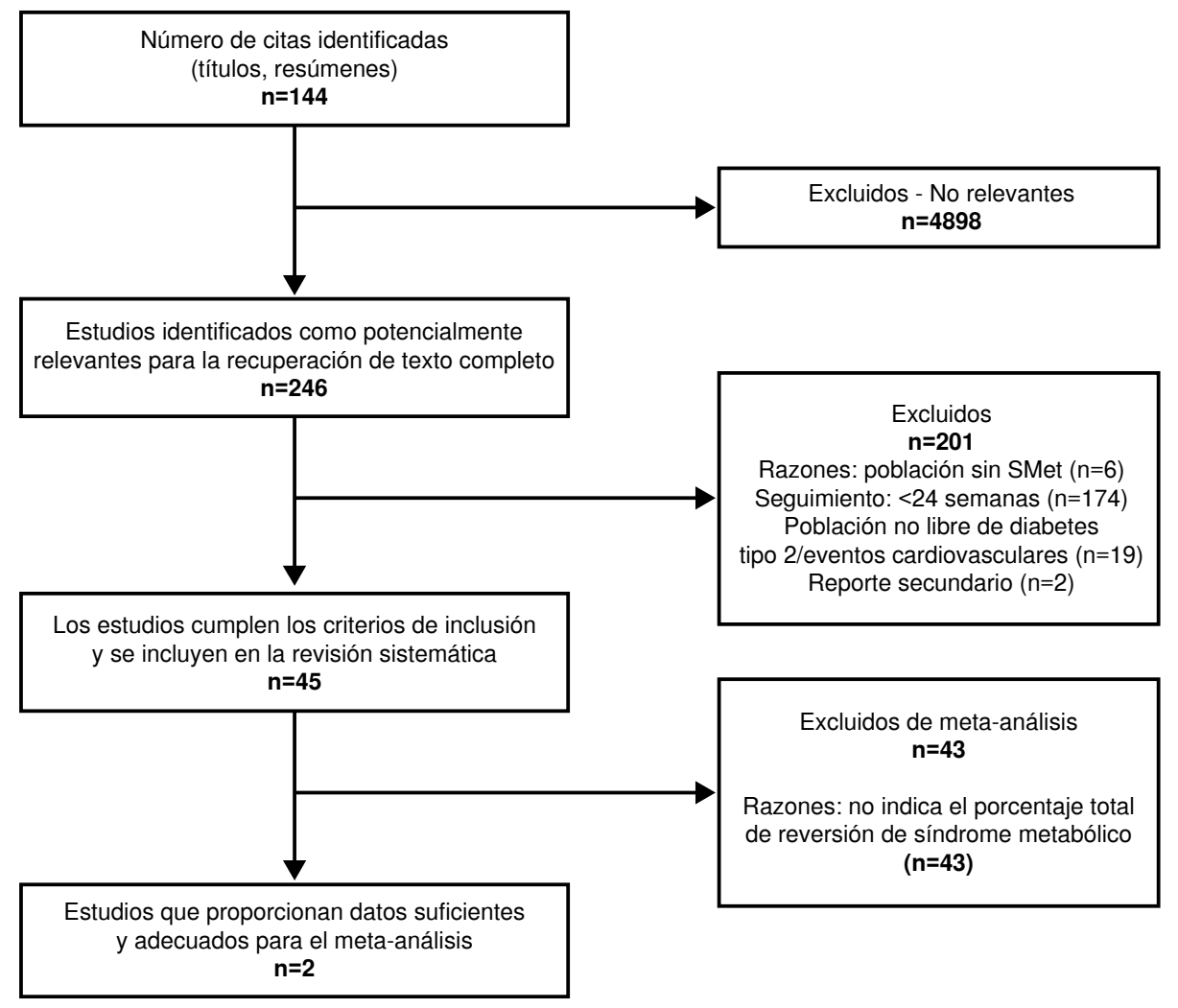

Figura 1. Clasificación de los estudios según los criterios de exclusión e inclusión (octubre de 2017) 


\section{Síntesis de la evidencia}

En la búsqueda de artículos posteriores al estudio de base (12), se encontraron dos estudios con datos suficientes para ser incluidos en el metaanálisis. Dadas las características de la 'evidencia', es necesario comparar los métodos de síntesis de la información; en este caso, se evaluó un modelo asumiendo efectos fijos y efectos aleatorios no correlacionados. Asimismo, dado que la 'evidencia' no se presentó vinculada, es decir, no todas las comparaciones posibles entre los tratamientos se encontraban en el mismo estudio clínico, la estimación de la OR debe basarse en el cálculo de las comparaciones que no se han hecho directamente en todos los estudios.

A este tercer tipo de modelo se le denomina 'método de comparaciones mixtas de tratamientos', ya que se basa en estimaciones directas e indirectas según la disponibilidad de los datos $(15,16)$. También se le conoce como metaanálisis de múltiple tratamiento (Multiple Treatment Meta-Analysis) o metaanálisis de redes (Network Meta-Analysis) (13,17). Para analizar este aspecto, se puede plantear un análisis geométrico a partir de anotar una red de información o de tratamientos. Las ventajas de este enfoque analítico es que permite estimar la probabilidad de eventos sobre los cuales se requiere tomar una decisión crítica, a pesar de la escasez de 'evidencia' científica.

En todos los análisis, se utilizó la comparación por pares de cada una de las intervenciones: la de control, la relacionada con el estilo de vida o con los fármacos y la combinación de estas dos. En la compilación de ambos modelos, se incorporaron solo seis de los trece artículos del estudio de base y los dos artículos detectados en la búsqueda durante el periodo adicional de observación, esto, con la finalidad de mantener la relación en la red de tratamientos durante la convergencia de los modelos, por lo que la estimación de la OR correspondió a la síntesis de las medidas reportadas en ocho de los artículos.

\section{Red de tratamientos}

La red construida para este estudio se presenta en la figura 2 y se basa en la integración de la evidencia en las cuatro categorías comparadas en este análisis. Cada categoría conformó un tipo de tratamiento según el enfoque tecnológico.

El control se definió como el comparador y se refiere a aquellas intervenciones de base en las que se observó la probabilidad de que el síndrome metabólico se revirtiera en una persona. Las intervenciones diseñadas para mejorar los hábitos nutricionales con la dieta, se integraron en la categoría de estilo de vida conjuntamente con las propuestas de entrenamiento físico. Los tratamientos farmacológicos podían incluir los medicamentos contra la obesidad y los antidiabéticos.

Por último, se contempló la combinación de un tratamiento farmacológico con los esquemas de estilo de vida. En la figura 2, se aprecia la distribución de la 'evidencia' según el número de comparaciones directas halladas en los artículos, siendo el contraste entre el control y el estilo de vida la relación con mayor evidencia directa, seguida del tratamiento farmacológico comparado con un control. El binomio con menor 'evidencia' fue el de un control y la combinación de las intervenciones. En este caso, la red quedó completamente conectada, lo que garantizó la solidez de los resultados analíticos. 


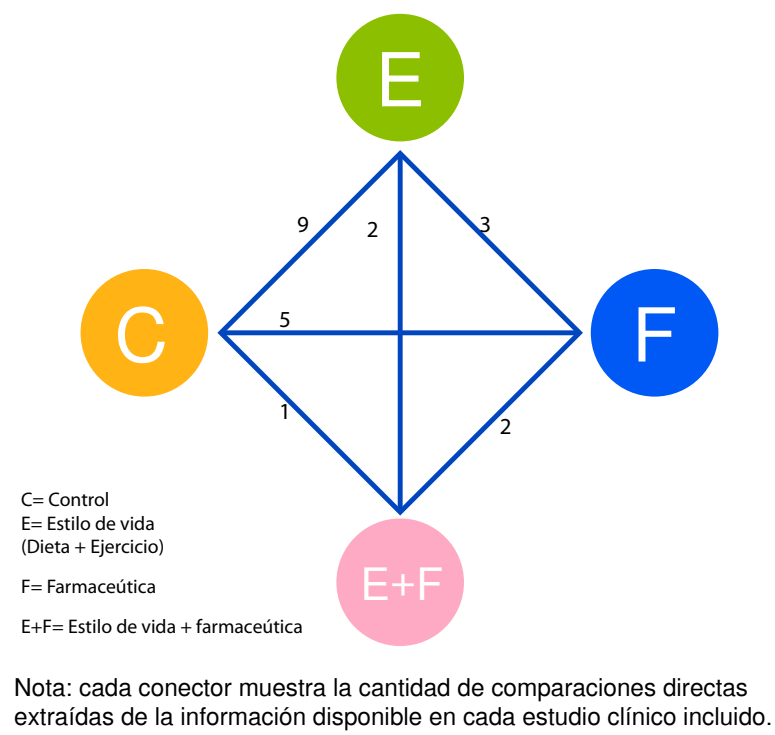

Figura 2. Red de evidencia de las intervenciones disponibles para la reversión del síndrome metabólico

\section{Análisis estadístico}

La OR de cada comparación requerida se calculó utilizando un enfoque de estadística bayesiana (18) mediante el programa de WinBUGS en cada uno de los modelos usados: efecto fijo, efecto aleatorio y comparaciones mixtas de tratamientos para considerar la correlación de las comparaciones. La plataforma de WinBUGS utiliza un algoritmo basado en el método de Monte Carlo con cadenas de Markov (Markov chain Monte Carlo, MCMC) y la herramienta de muestreo de Gibbs para estimar los parámetros estadísticos (media, mediana y desviación estándar) de una función de densidad posterior (19-22). Cada modelo se compiló con un desecho de las primeras 5.000 iteraciones y los parámetros reportados en este artículo se basan en la estimación ofrecida por la función de densidad posterior generada luego de 10.000 iteraciones.

El análisis de los modelos se hizo en la versión 14 de la plataforma de software de WinBUGS y el diagrama de bosque con el programa R (versión 3,1 ), en tanto que las tablas se formatearon con Excel. En la preparación de estos resultados, se siguió la guía PRISMA (23).

La selección de los estudios y la extracción de los datos estuvieron a cargo de un equipo de cinco investigadores independientes y se usaron diferentes estrategias para corroborar la exhaustividad de la estrategia de búsqueda y la detección de estudios, así como la calidad de la extracción de los datos, tal como se especificaron en las secciones anteriores.

\section{Resultados}

Se seleccionaron 45 artículos según los criterios de inclusión y exclusión. En la figura 1, se muestra la primera selección de 246 documentos. Tras revisar la prevalencia del síndrome metabólico antes de la intervención y después de ella, solamente en dos artículos $(24,25)$ se utilizó un enfoque binomial para clasificar el síndrome, es decir, reportaron si cumplían o no con los criterios para su diagnóstico. 
En los 15 estudios que hicieron parte de la revisión sistemática, se incluyeron los siguientes tratamientos implementados y evaluados por separado o de manera combinada: (i) asesoramiento dietético individualizado e intensivo; (ii) sesiones supervisadas de ejercicio; (iii) consejos de ejercicios; (iv) metformina; (v) rosiglitazona; (vi) atorvastatina; (vii) pravastatina; (viii) lovastatina; (ix) fenofibrato; $(x)$ sibutramina, y (xi) rimonabant.

Se encontraron ocho estudios centrados únicamente en la efectividad de las intervenciones relacionadas con el estilo de vida (uno, solo dieta; dos, solo ejercicios; cuatro, dietas combinadas con ejercicio, y uno, dieta, educación y ejercicio). En cuatro estudios, se compararon la efectividad de las intervenciones farmacológicas y, en tres de ellos, se combinaron intervenciones farmacológicas con las relacionadas con el estilo de vida. En doce de los estudios se hizo análisis de subgrupos, y ocho de estos fueron publicados posteriormente en documentos separados (post hoc). Los artículos revisados se habían publicado en los 11 años anteriores y los estudios se llevaron a cabo en Grecia, Noruega, Italia, Holanda, Estados Unidos, China y Corea.

Se utilizó la definición del NCEP como criterio de inclusión de los estudios en el metaanálisis. Con respecto a la duración de los estudios, se tuvieron en cuenta períodos de seguimiento a partir de 24 semanas y hasta cuatro años. La edad media de los participantes y el índice de masa corporal, variaron de 44 a 70 años y de 25,6 a $39 \mathrm{~kg} / \mathrm{m}^{2}$, respectivamente. El porcentaje de hombres fluctuó entre 21 y $100 \%$. Tal como en el estudio de base, se definió la reversión del síndrome metabólico según la proporción de individuos con diagnóstico positivo en el estado inicial del estudio y cuya condición cambió en el curso del seguimiento.

Los dos estudios informaron adecuadamente sobre los criterios de inclusión en el análisis y los porcentajes de reversión del síndrome metabólico, pero solo en uno se reportaron las OR de la reversión para el tratamiento y el correspondiente intervalo de confianza para la estimación.

Como se constata en la red de tratamientos presentada en la figura 2, en los artículos incluidos en el análisis se encontraron cuatro diferentes comparaciones entre los tratamientos. En el cuadro 1, se presentan las características de los estudios revisados, y se registran los autores y el año de publicación, el país, el tamaño de la muestra, el tipo de intervención, y las características de los participantes y del diseño del estudio, así como las definiciones utilizadas.

Los contrastes más frecuentes (nueve artículos), se hicieron entre el estilo de vida y el grupo de control: Esposito, et al., 2004 (29); Stewart, et al., 2005 (36); Orchard, et al., 2005 (33); Villareal, et al., 2006 (38); Ramachandran, et al., 2006 (35); Anderssen, et al., 2007 (26); Bo, et al., 2007 (28); Yoo, et al., 2012 (24) y Siu, et al., 2015 (25). Además, en cinco artículos se compararon tratamientos con esquemas farmacológicos versus un grupo control: Orchard, et al., 2005 (33); van Gaal, et al., 2005 (37); Geluk, et al., 2005 (31); y Esposito, et al., 2006 (30), y Ramachandran, et al., 2006 (35).

Otro de los contrastes incluidos en los estudios fue entre la combinación de las intervenciones relacionadas con el estilo de vida y los tratamientos farmacológicos, la cual se registró en tres artículos: Orchard, et al., 2005 (33), Ramachandran, et al., 2006 (35) y Phelan, et al., 2007 (34). 
Cuadro 1. Detalles de todos los estudios incluidos en la revisión sistemática y descripción de cada grupo de intervención

\begin{tabular}{|c|c|c|c|c|c|c|c|c|c|c|c|c|c|}
\hline Autor & Año & Definición & País & $\begin{array}{c}\text { Tamaño } \\
\text { de la } \\
\text { muestra }\end{array}$ & $\begin{array}{c}\text { Tamaño de } \\
\text { muestra } \\
\text { con MetS }\end{array}$ & $\begin{array}{c}\text { Foco de } \\
\text { intervención }\end{array}$ & $\begin{array}{c}\text { Edad, } \\
\text { media } \\
\text { (DE) }\end{array}$ & $\begin{array}{c}\text { Masculino } \\
\%\end{array}$ & $\begin{array}{c}\text { IMC, media } \\
\text { (DE) } \\
\text { kg/m2 }\end{array}$ & Etnia \% & $\begin{array}{l}\text { Periodo de } \\
\text { seguimiento }\end{array}$ & $\begin{array}{l}\text { Reversión } \\
\text { del síndrome } \\
\text { metabólico }\end{array}$ & $\begin{array}{c}\text { Otros } \\
\text { resultados }\end{array}$ \\
\hline $\begin{array}{l}\text { Anderssen, et } \\
\text { al. [26] }\end{array}$ & 2007 & IDF & Noruega & 188 & 137 & $\begin{array}{l}\text { Estilo de } \\
\text { vida (dieta } \\
\text { y ejercicio } \\
\text { supervisado) }\end{array}$ & $45(2,5)$ & 100 & 29 & NR & 1 año & Sí & - \\
\hline $\begin{array}{l}\text { Athyros, et al. } \\
\text { [27] }\end{array}$ & 2005 & NCEP & Grecia & 300 & 300 & $\begin{array}{l}\text { Atorvastina y } \\
\text { fenofibrato }\end{array}$ & NR & 63 & 31 & NR & 1 año & Sí & - \\
\hline Bo, et al. [28] & 2007 & NCEP & Italia & 335 & 239 & $\begin{array}{l}\text { Estilo de } \\
\text { vida (dieta } \\
\text { y ejercicio } \\
\text { supervisado) }\end{array}$ & $56^{*}$ & $42^{*}$ & 30 & NR & 1 año & Sí & $\begin{array}{l}\text { Incidencia } \\
\text { de T2DM }\end{array}$ \\
\hline $\begin{array}{l}\text { Esposito, et } \\
\text { al. [29] }\end{array}$ & 2004 & NCEP & Italia & 180 & 180 & $\begin{array}{l}\text { Estilo de vida } \\
\text { (dieta) }\end{array}$ & 44 & 55 & 28 & NR & 2 años & Sí & - \\
\hline $\begin{array}{l}\text { Esposito, et } \\
\text { al. [30] }\end{array}$ & 2006 & NCEP & Italia & 100 & 100 & Rosigilitazona & $46(4,5)$ & 54 & 28 & NR & 1 año & Sí & - \\
\hline $\begin{array}{l}\text { Geluk, et al. } \\
\text { [31] }\end{array}$ & 2005 & NCEP & Holanda & 864 & 228 & Pravastatina & $55(11)^{\star *}$ & $70^{* *}$ & 29 & $\begin{array}{l}96 \% \\
\text { Caucásico }\end{array}$ & 4 años & Sí & $\begin{array}{l}\text { Evento } \\
\text { CHD o } \\
\text { muerte }\end{array}$ \\
\hline $\begin{array}{l}\text { Johnson, et } \\
\text { al. [32] }\end{array}$ & & NCEP & $\begin{array}{l}\text { Estados } \\
\text { Unidos }\end{array}$ & 334 & 69 & $\begin{array}{l}\text { Estilo de vida } \\
\text { (ejercicio } \\
\text { supervisado) }\end{array}$ & $53(7)^{*}$ & $53^{*}$ & 30 & NR & 8 meses & Sí & - \\
\hline $\begin{array}{l}\text { Orchard, et al. } \\
\text { [33] }\end{array}$ & 2005 & NCEP & $\begin{array}{l}\text { Estados } \\
\text { Unidos }\end{array}$ & 3324 & 1711 & $\begin{array}{l}\text { Estilo de } \\
\text { vida (dieta y } \\
\text { consejería } \\
\text { deportiva) } \\
\text { Metformina }\end{array}$ & $51(10,7)^{*}$ & $32^{*}$ & 34 & $\begin{array}{l}55 \% \\
\text { Blanco, } \\
20 \% \text { Af- } \\
\text { Am, } 16 \% \\
\text { Hip, } 9 \% \\
\text { otro }\end{array}$ & 3 años & Sí & - \\
\hline $\begin{array}{l}\text { Phelan, et al. } \\
\text { [34] }\end{array}$ & 2007 & NCEP & $\begin{array}{l}\text { Estados } \\
\text { Unidos }\end{array}$ & 224 & 78 & $\begin{array}{l}\text { Estilo de } \\
\text { vida (dieta y } \\
\text { consejería } \\
\text { deportiva) } \\
\text { Sibutramina }\end{array}$ & $48(9,9)^{*}$ & 37 & 38 & $\begin{array}{l}80 \% \\
\text { Blancos } \\
18 \% \text { Af- } \\
\text { Am, } 3 \% \\
\text { Hisp. }\end{array}$ & 1 año & Sí & - \\
\hline $\begin{array}{l}\text { Ramachandran, } \\
\text { et al. [35] }\end{array}$ & 2006 & OMS & India & 521 & 233 & $\begin{array}{l}\text { Estilo de } \\
\text { vida (dieta y } \\
\text { consejería } \\
\text { deportiva) } \\
\text { Metformina }\end{array}$ & $46^{*}$ & $79^{*}$ & 26 & $\begin{array}{l}\text { Indio } \\
\text { asiático } \\
\text { nativo }\end{array}$ & 3 años & Sí & $\begin{array}{l}\text { Incidencia } \\
\text { de T2DM }\end{array}$ \\
\hline $\begin{array}{l}\text { Stewart, et al. } \\
{[36]}\end{array}$ & 2005 & NCEP & $\begin{array}{l}\text { Estados } \\
\text { Unidos }\end{array}$ & 115 & 44 & $\begin{array}{l}\text { Estilo de } \\
\text { vida (dieta } \\
\text { y ejercicio } \\
\text { supervisado) }\end{array}$ & $64(5,7)^{*}$ & $49^{*}$ & 30 & $\begin{array}{l}87 \% \\
\text { Blancos, } \\
11 \% \text { Af- } \\
\text { Am, } 2 \% \text {, } \\
\text { otros }\end{array}$ & 26 semanas & Sí & - \\
\hline $\begin{array}{l}\text { van Gaal, et } \\
\text { al. [37] }\end{array}$ & 2005 & NCEP & $\begin{array}{l}\text { Europa- } \\
\text { Estados } \\
\text { Unidos }\end{array}$ & 1507 & 564 & Rimonabant & $45^{*}$ & $21^{*}$ & 36 & $\begin{array}{l}94 \% \\
\text { Blanco }\end{array}$ & 1 año & Sí & - \\
\hline $\begin{array}{l}\text { Villareal, et al. } \\
\text { [38] }\end{array}$ & 2006 & NCEP & $\begin{array}{l}\text { Estados } \\
\text { Unidos }\end{array}$ & 27 & 24 & $\begin{array}{l}\text { Estilo de } \\
\text { vida (dieta } \\
\text { y ejercicio } \\
\text { supervisado) }\end{array}$ & $70^{*}$ & $33^{*}$ & 39 & $\begin{array}{l}85 \% \\
\text { Blanco }\end{array}$ & 26 semanas & Sí & - \\
\hline Siu, et al. [25] & 2015 & NCEP & China & 182 & 76 & $\begin{array}{l}\text { Estilo de vida } \\
\text { (ejercicio } \\
\text { supervisado) }\end{array}$ & $56(9,1)$ & 32 & -- & Asiático & 1 año & Sí & - \\
\hline Yoo, et al. [24] & 2012 & NCEP & Corea & 195 & 108 & $\begin{array}{l}\text { Estilo de vida } \\
\text { (educación, } \\
\text { dieta y } \\
\text { ejercicio } \\
\text { supervisado) }\end{array}$ & $65(3,38)$ & 38 & $25.87(2.3)$ & Asiático & 6 meses & Sí & - \\
\hline
\end{tabular}

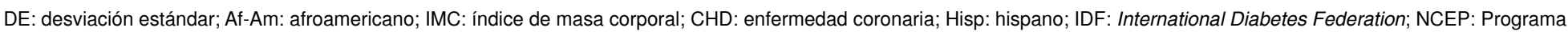
Nacional de Educación sobre el Colesterol; NR, no reportado; T2DM: diabetes mellitus de tipo 2; OMS: Organización Mundial de la Salud

* Los datos indicados corresponden a la prueba principal, no estaban disponibles para el subgrupo.

** Datos únicamente de edad, etc., incluida la diabetes mellitus. Fuente: Base de datos EMBASE y Medline, consultadas en octubre de 2017 
Asimismo, en dos artículos se comparó la combinación de una intervención relacionada con el estilo de vida y el tratamiento farmacológico con cada una de estas intervenciones por separado: Ramachandran, et al., 2007 (35) y Phelan, et al., 2007 (34). Por último, solamente en un artículo se compararon la combinación del tratamiento farmacológico y la intervención relacionada con el estilo de vida, versus el grupo control. Cabe mencionar que más de un tipo de comparación puede aparecer en un solo artículo.

En el cuadro 1, se presentan también la categorización de cada grupo de intervención y el número de casos de reversión del síndrome metabólico por tipo de intervención. Al agregar los dos estudios publicados después del estudio de base (12), el metaanálisis incluyó los datos de 4.291 participantes con el síndrome, y los resultados que evidenciaron los beneficios de las intervenciones en el estilo de vida y las farmacológicas.

En la figura 3, se presenta el diagrama de bosque (forest plot) de los efectos de las diferentes intervenciones empleadas en los estudios analizados en la reversión del síndrome metabólico y el efecto combinado estimado con modelos de efectos fijos y efectos aleatorios no correlacionados. En ella, se aprecia también que, en contraste con los grupos de control, las intervenciones en el estilo de vida tienen más probabilidades de revertir el síndrome que las farmacológicas. Además, con respecto al modelo de efectos fijos, el efecto combinado de las intervenciones en el estilo de vida comparadas con los grupos de control es mayor $(\mathrm{OR}=2,52$; $\left.I C_{95 \%}=2,06-3,05\right)$ que el de las farmacológicas $\left(O R=1,51 ; I C_{95 \%}=1,19-1,90\right)$, así como en el caso de la combinación de ambas $\left(\mathrm{OR}=1,14 ; \mathrm{IC}_{95 \%}=0,47-\right.$ $2,72)$. En cuanto al modelo de efectos aleatorios no correlacionados, el efecto combinado de las intervenciones en el estilo de vida se contrajo más (efectos aleatorios no correlacionados, $\mathrm{OR}=2,61 ; \mathrm{IC}_{95 \%}=1,00-5,47$ ) que en las farmacológicas (efectos aleatorios no correlacionados, $O R=3,39 ; \mathrm{IC}_{95 \%}=0,81$ $9,99)$ en comparación con los grupos de control.

Al comparar directamente el efecto entre ambas intervenciones, las OR de reversión del síndrome metabólico al intervenir en el estilo de vida disminuyeron en $\mathbf{4 0} \%$ en comparación con las intervenciones farmacológicas según los resultados del modelo de efectos fijos (OR=0,60; $\left.I C_{95 \%}=0,47-0,75\right)$. Por otra parte, según el modelo de efectos aleatorios no correlacionados, la probabilidad de reversión mediante las intervenciones en el estilo de vida, fue 59 \% mayor que la de las intervenciones farmacológicas.

En el cuadro 2, se presenta la comparación entre el método de efectos fijos y el método de efectos aleatorios no correlacionados, y las intervenciones comparadas con los tratamientos. Se observa que, en ambos modelos, las intervenciones relacionadas con el estilo de vida y la utilización de un fármaco obtuvieron OR mayores de 1 con respecto al grupo de control, lo que evidencia que son efectivas para revertir el diagnóstico de síndrome metabólico. Cabe destacar que el modelo de efectos aleatorios no correlacionados contradice lo obtenido con el de efectos fijos con respecto al cambio del estilo de vida en conjunto con un fármaco; este tratamiento conjunto fue beneficioso para revertir el síndrome al usar el modelo de efectos aleatorios no correlacionados y hacer la comparación con el grupo control.

Se utilizó el criterio de información de desviación (deviance information criterion) para elegir entre ambos modelos mediante la evaluación de la bondad de ajuste y se lo valoró como método de validación de los resultados. Como se observa en el cuadro 2, el modelo de efectos aleatorios presentó 


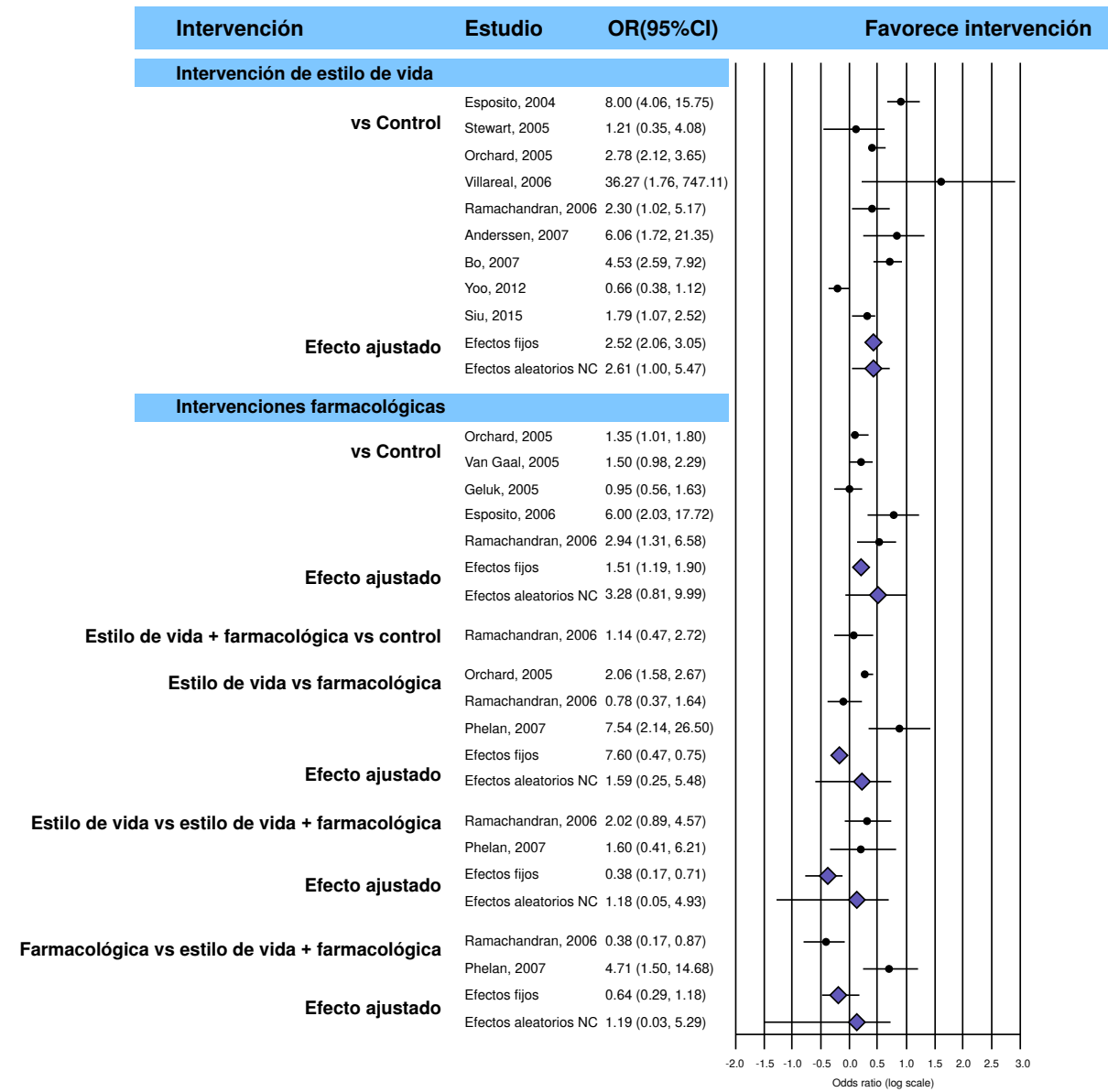

Figura 3. Efectos de las diferentes intervenciones en la reversión del síndrome metabólico (razón de momios, OR)

Cuadro 2. Resultados de las comparaciones de tratamientos mediante modelos de efectos fijos y de efectos aleatorios no correlacionados

\begin{tabular}{|c|c|c|c|c|}
\hline \multirow{2}{*}{ Comparación } & \multicolumn{2}{|c|}{ Efectos fijos } & \multicolumn{2}{|c|}{ Efectos aleatorios no correlacionados } \\
\hline & OR & $\left(\mathrm{IC}_{95} \%\right)$ & OR & $\left(\mathrm{IC}_{95} \%\right)$ \\
\hline Estilo de vida Vs. control & 2,52 & $(2,06-3,05)$ & 2,61 & $(1,00-5,47)$ \\
\hline Farmacológica Vs. control & 1,51 & $(1,19-1,90)$ & 3,39 & $(0,81-9,99)$ \\
\hline Farmacológica y estilo de vida Vs. control & 0,96 & $(0,43-1,79)$ & 2,31 & $(0,13-10,31)$ \\
\hline Farmacológica Vs. estilo de vida & 0,60 & $(0,47-0,75)$ & 1,59 & $(0,25-5,48)$ \\
\hline Farmacológica y estilo de vida Vs. estilo de vida & 0,38 & $(0,17-0,71)$ & 1,18 & $(0,05-4,93)$ \\
\hline Farmacológica + estilo de vida Vs. farmacológica & 0,64 & $(0,29-1,18)$ & 1,19 & $(0,03-5,29)$ \\
\hline DIC & 167,57 & 125,74 & & \\
\hline
\end{tabular}

DIC: Deviation information criterion

una mejor bondad de ajuste, debido a que el indicador de desviación para este modelo resultó ser menor $(125,74)$ en comparación con el obtenido con el modelo de efectos fijos. Se fijó una distribución uniforme para configurar a priori un parámetro de la heterogeneidad de los estudios, por lo que se asumió que los errores de precisión de los estudios seguían un patrón uniforme.

En el cuadro 3, se presentan las OR de los modelos del artículo de referencia (metaanálisis directo y análisis de comparación mixta de tratamientos), comparadas con los modelos estimados (efectos fijos y efectos aleatorios no correlacionados), así como las estimaciones obtenidas al modelar un ajuste según la correlación entre las comparaciones, con lo que se consideraron las diferencias directas e indirectas para cada contraste 
Cuadro 3. Comparación de los resultados del artículo de referencia con los de los actualizados

\begin{tabular}{|c|c|c|c|c|c|c|c|c|}
\hline \multirow[t]{2}{*}{ Comparación } & \multicolumn{2}{|c|}{ Metaanálisis directo } & \multicolumn{2}{|c|}{$\begin{array}{c}\text { Análisis de } \\
\text { comparaciones mixtas } \\
\text { de tratamientos }\end{array}$} & \multicolumn{2}{|c|}{$\begin{array}{c}\text { Comparaciones } \\
\text { mixtas de tratamientos } \\
\text { actualizadas }\end{array}$} & \multicolumn{2}{|c|}{$\begin{array}{c}\text { Efectos aleatorios } \\
\text { no correlacionados } \\
\text { actualizados }\end{array}$} \\
\hline & OR & $\left(\mathrm{IC}_{95} \%\right)$ & OR & $\left(\mathrm{IC}_{95} \%\right)$ & OR & $\left(\mathrm{IC}_{95} \%\right)$ & OR & $\left(\mathrm{IC}_{95} \%\right)$ \\
\hline Estilo de vida Vs. control & 3,81 & $(2,47-5,88)$ & 4,43 & $(2,36-9,16)$ & 6,06 & $(2,55-14,23)$ & 2,606 & $(1-5,47)$ \\
\hline Farmacológica Vs. control & 1,59 & $(1,04-2,45)$ & 1,73 & $(0,73-3,80)$ & 3,13 & $(1,13-7,71)$ & 3,387 & $(0,81-9,99)$ \\
\hline Farmacológica y estilo de vida Vs. control & 1,14 & $(0,48-2,72)$ & 2,16 & $(0,63-8,29)$ & 2.27 & $(0,26-8,93)$ & 2,313 & $(0,13-10,31)$ \\
\hline Farmacológica Vs. estilo de vida & 0,49 & $(0,20-1,24)$ & 0,39 & $(0,16-0,90)$ & 0,58 & $(0,16-1,45)$ & 1,586 & $(0,25-5,48)$ \\
\hline Farmacológica y estilo de vida Vs. estilo de vida & 0,53 & $(0,26-1,06)$ & 0,49 & $(0,14-1,74)$ & 0,41 & $(0,04-1,53)$ & 1,177 & $(0,05-4,93)$ \\
\hline Farmacológica y estilo de vida Vs. farmacológica & 1,31 & $(0,11-15,13)$ & 1,25 & $(0,36-4,49)$ & 0,84 & $(0,08-3,16)$ & 1,185 & $(0,03-5,29)$ \\
\hline
\end{tabular}

Nota: El modelo de efectos aleatorios no correlacionados se compara con el metaanálisis directo del artículo de Dunkley, et al., 2012.

requerido. Se observó que, en ambos casos, en el artículo de referencia, las intervenciones (estilo de vida, fármacos, y estilo de vida y fármacos en conjunto) presentaban ventajas mayores de 1, en comparación con el control, lo que confirmó el efecto positivo sobre la reversión del síndrome metabólico. Sin embargo, los intervalos de credibilidad para las intervenciones en el estilo de vida no incluyeron el 1, lo cual demostró la superioridad de este tipo de tratamiento comparado con los farmacológicos.

Por último, en el mismo cuadro 3, se reportan las OR de diez estudios resultantes del análisis de redes de evidencia y de las comparaciones mixtas de tratamientos a partir de modelos bayesianos. Se analizó la sensibilidad para valorar la bondad de ajuste del modelo completo en la estimación de las OR actualizadas, utilizando el método de comparaciones mixtas de tratamientos. El análisis consistió en excluir el estudio con los resultados más extremos en la efectividad, es decir, el de Villareal, et al. (38), en el cual el grupo de control no registró reversión alguna. El criterio de información de desviación del modelo en los diez estudios resultó en un valor de 126,64, pero al calcularlo eliminando los datos del estudio de Villareal, este indicador se elevó a 130,47.

\section{Discusión}

Esta síntesis de la información permitió determinar el tipo de intervención más efectiva para revertir el síndrome. En la síntesis de Dunkley, et al. (12), los hallazgos no fueron suficientes para demostrar las diferencias entre los tratamientos farmacéuticos y las intervenciones en el estilo de vida; sin embargo, al añadir los estudios publicados en el 2012 por Yoo, et al. (24) y Siu, et al. (25), se encontró una efectividad significativa de las estrategias basadas en el ejercicio, la dieta y la educación.

Con base en los datos recopilados sobre la red de tratamientos relacionados con la posibilidad de reversión, se pudo trazar una trayectoria histórica en torno a los datos disponibles. Hay claridad en cuanto a que ha habido un cambio en la manera de reportar el producto de la estimación reportada para la efectividad de las intervenciones y sus efectos en personas con síndrome metabólico. Se encontraron solo dos estudios nuevos con un reporte binario del síndrome. Se excluyeron los estudios que involucraban una jerarquía mucho más compleja, al considerar los efectos en los diferentes componentes del síndrome, pues transformaban la variable de respuesta de una distribución binomial a una distribución multivariada, con una amplia gama de categorías posibles en las que se localizarían los individuos que cumplen con los criterios de clasificación del síndrome metabólico. 
La comparación de los resultados de la red de tratamientos presentada en la figura 2 con la estimación actualizada de las OR mediante comparaciones mixtas de tratamientos, permitió valorar un acervo de información mucho más abundante, estimar las medidas con intervalos de credibilidad mucho más sólidos y observar de manera más detallada el tipo de patrón que se construye conforme se agrega más información relevante.

El contraste con mayor información se obtiene al comparar el estilo de vida con algún otro factor basal que sirva para conformar un grupo de control y favorezca la comparación entre los tratamientos. En este caso, se encontraron nueve estimaciones directas en los estudios analizados, después de excluir los artículos que no reportaron ningún control.

Al integrar la información indirecta de esta comparación, la probabilidad bayesiana de revertir el síndrome fue seis veces mayor cuando se involucró a las personas en procesos de aprendizaje general sobre su nutrición y la forma de ejercitarse. El elemento de comparación generalizado fueron las intervenciones de base en las que se les dio a los participantes algún consejo general sobre hábitos saludables o se les dijo que continuaran con los que tenían en el momento del estudio.

También se demuestra que las farmacoterapias tienen un efecto importante en la reversión del síndrome; esta comparación fue la segunda más informada, con cinco comparaciones directas obtenidas de la información utilizada. Sin embargo, en este caso, la intensidad del efecto de la intervención farmacéutica se reduce en $50 \%$ con respecto a la intervención en el estilo de vida. Por lo tanto, el tratamiento basado en fármacos ofrece una efectividad tres veces mayor de revertir un diagnóstico del síndrome, considerando una variabilidad de la OR entre 1,13 y 7,71. Esto podría estar vinculado con el hecho de que no necesariamente se opta por los tratamientos farmacológicos para el manejo de los componentes del síndrome metabólico.

En cuanto a las estimaciones de la comparación basal de la intervención combinada, se encontró un efecto elevado bastante apreciable; sin embargo, dado que esta comparación fue la menos reportada, con una sola comparación directa, el intervalo de credibilidad incluyó la unidad. Por lo tanto, sería necesario agregar más información para valorar si la relación se mantiene en una efectividad más dispersa o si se confirma la necesidad de descartarla.

Ahora bien, esta es una comparación que, en la práctica clínica, siempre se va a dar en ciertos grupos de individuos con condiciones que podrían requerir medicación, como la hipertensión arterial sistémica. Es importante anotar que esta intervención debería estudiarse dadas las posibles interacciones. Las demás comparaciones no resultaron significativas, ya que, a pesar de tener más datos, los intervalos mantuvieron la amplitud que incluye la unidad, o de efecto nulo.

En cuanto a las limitaciones de este estudio, está la necesidad de establecer una red de tratamientos mucho más específica y explorar los detalles de las intervenciones, para posibilitar diseños innovadores en la gestión de las políticas de salud pública. Además, debería hacerse un análisis de sesgo de publicación, con el fin de observar si estas estimaciones merecen un nuevo ajuste. 
En conclusión, al comparar los resultados de estos 15 artículos con el de Dunkley, et al., el cual incluyó 13 estudios (12), se demostró que, en ambos casos, el uso de cualquiera de las intervenciones o tratamientos ayudó a revertir el síndrome metabólico. Sin embargo, la actualización permite demostrar una superioridad en la efectividad de las intervenciones por estilo de vida.

\section{Referencias}

1. O'neill S, O'driscoll L. Metabolic syndrome: A closer look at the growing epidemic and its associated pathologies. Obes Rev. 2015;16:1-12. https://doi.org/10.1111/obr.12229

2. Balkau B, Charles MA, Drivsholm T, Borch-Johnsen K, Wareham N, Yudkin JS, et al. Frequency of the WHO metabolic syndrome in European cohorts, and an alternative definition of an insulin resistance syndrome. Diabet Metab. 2002;28:364-76.

3. Cameron AJ, Shaw JE, Zimmet PZ. The metabolic syndrome: Prevalence in worldwide populations. Endocrinol Metab Clin North Am. 2004;33:351-75. https://doi.org/10.1016/j.ecl.2004.03.005

4. Aguilar-Salinas CA, Rojas R, Gómez-Pérez FJ, Valles V, Ríos-Torres JM, Franco A, et al. Analysis of the agreement between the World Health Organization criteria and the National Cholesterol Education Program-III definition of the metabolic syndrome: Results from a population-based survey. Diabetes Care. 2003;26:1635. https://doi.org/10.2337/diacare.26.5.1635

5. Villegas R, Perry IJ, Creagh D, Hinchion R, O'Halloran D. Prevalence of the metabolic syndrome in middle-aged men and women. Diabetes Care. 2003;26:3198-9. https://doi.org/10.2337/diacare.26.11.3198-a

6. Ford ES, Giles WH, Dietz WH. Prevalence of the metabolic syndrome among US adults: Findings from the Third National Health and Nutrition Examination Survey. JAMA 2002;287:356-9. https://doi:10.1001/jama.287.3.356

7. Gupta A, Gupta R, Sarna M, Rastogi S, Gupta VP, Kothari K. Prevalence of diabetes, impaired fasting glucose and insulin resistance syndrome in an urban Indian population. Diab Res Clin Pract. 2003;61:69-76. https://doi.org/10.1016/S0168-8227(03)00085-8

8. Zimmet P, Shaw J, Alberti KG. Preventing type 2 diabetes and the dysmetabolic syndrome in the real world: A realistic view. Diabet Med. 2003;20:693-702. https://doi.org/10.1046/j.1464-5491.2003.01052.x

9. Gami AS, Witt BJ, Howard DE, Erwin PJ, Gami LA, Somers VK, et al. Metabolic syndrome and risk of incident cardiovascular events and death: A systematic review and meta-analysis of longitudinal studies. J Am Coll Cardiol. 2007;49:403-14. https://doi.org/10.1016/j.jacc.2006.09.032

10. Mottillo S, Filion KB, Genest J, Joseph L, Pilote L, Poirier P, et al. The metabolic syndrome and cardiovascular risk: A systematic review and meta-analysis. J Am Coll Cardiol. 2010;56:1113-32. https://doi.org/10.1016/j.jacc.2010.05.034

11. Ford ES, Li C, Sattar N. Metabolic syndrome and incident diabetes: Current state of the evidence. Diabetes Care. 2008;31:1898-904. https://doi.org/10.2337/dc08-0423

12. Dunkley AJ, Charles K, Gray LJ, Camosso-Stefinovic J, Davies MJ, Khunti K. Effectiveness of interventions for reducing diabetes and cardiovascular disease risk in people with metabolic syndrome: Systematic review and mixed treament comparison meta-analysis. Diabetes Obes Metab. 2012;14:616-25. https://doi.org/10.1111/j.1463-1326.2012.01571.x

13. García-Perdomo HA, Tobías A. Metanálisis en red: comparaciones indirectas y mixtas, un nuevo método al servicio de la epidemiología clínica y la salud pública. Rev Perú Med Exp Salud Pública. 2016;33:149-53. https://doi.org/10.17843/rpmesp.2016.331.1889

14. Higgins J, Green S. Cochrane Handbook for Systematic Reviews of Interventions, Version 5.1.0. The Cochrane Collaboration; 2011. Disponible en: https://handbook-5-1.cochrane.org/

15. Bucher H, Guyatt GH, Griffith L, Walter SD. The results of direct and indirect treatment comparisons in meta-analysis of randomized controlled trials. J Clin Epidemiol. 1997;50:683-91. https://doi.org/10.1016/S0895-4356(97)00049-8

16. Coleman Cl, Phung OJ, Cappelleri JC, Baker WL, Kluger J, White CM, et al. Use of mixed treatment comparisons in systematic reviews. 2012. Rockville, MD: Agency for Healthcare Research and Quality; 2012.

17. Salanti G, Higgins JP, Ades AE, loannidis JP. Evaluation of networks of randomized trials. Stat Methods Med Res. 2008;17:279-301. https://doi.org/10.1177/0962280207080643 
18. Gutiérrez-Pulido H, Gutiérrez-González P. Fundamentos y aplicaciones de la estadística Bayesiana. México: Universidad de Guadalajara; 2013.

19. Sutton AJ, Abrams KR. Bayesian methods in meta-analysis and evidence synthesis. Stat Methods Med Res. 2001;10:277-303. https://doi.org/10.1177/096228020101000404

20. Song F, Altman DG, Glenny AM, Deeks JJ. Validity of indirect comparison for estimating efficacy of competing interventions: Empirical evidence from published meta-analyses. BMJ. 2003;326:472. https://doi.org/10.1136/bmj.326.7387.472

21. Lam SK, Owen A. Combined resynchronisation and implantable defibrillator therapy in left ventricular dysfunction: Bayesian network meta-analysis of randomised controlled trials. BMJ. 2007;335:925. https://doi.org/10.1136/bmj.39343.511389.BE

22. Cooper NJ, Sutton AJ, Morris D, Ades AE, Welton NJ. Addressing between-study heterogeneity and inconsistency in mixed treatment comparisons: Application to stroke prevention treatments in individuals with non-rheumatic atrial fibrillation. Stat Med. 2009;28:1861-81. https://doi.org/10.1002/sim.3594

23. Moher D, Liberati A, Tetzlaff J, Altman DG. Preferred reporting items for systematic reviews and meta-analyses: The PRISMA statement. Ann Intern Med. 2009;151:264-9. https://doi.org/10.7326/0003-4819-151-4-200908180-00135

24. Yoo S, Kim H, Cho HI. Improvements in the metabolic syndrome and stages of change for lifestyle behaviors in Korean older adults. Osong Public Health Res Pespect. 2012;3:85-93. https://doi.org/10.1016/j.phrp.2012.04.007

25. Siu PM, Angus PY, Benzie IF, Woo J. Effects of 1-year yoga on cardiovascular risk factors in middle-aged and older adults with metabolic syndrome: A randomized trial. Diabetol Metab Syndr. 2015;7:40. https://doi.org/10.1186/s13098-015-0034-3

26. Anderssen S, Carroll S, Urdal P, Holme I. Combined diet and exercise intervention reverses the metabolic syndrome in middle-aged males: Results from the Oslo Diet and Exercise Study. Scand J Med Sci Sports. 2007;17:687. https://doi.org/10.1111/j.16000838.2006.00631.x

27. Athyros VG, Mikhailidis DP, Papageorgiou AA, Didangelos TP, Peletidou A, Kleta D, et al. Targeting vascular risk in patients with metabolic syndrome but without diabetes. Metabolism. 2005;54:1065-74. https://doi.org/10.1016/j.metabol.2005.03.010

28. Bo S, Ciccone G, Baldi C, Benini L, Dusio F, Forastiere G, et al. Effectiveness of a lifestyle intervention on metabolic syndrome. A randomized controlled trial. J Gen Intern Med. 2007;22:1695-703. https://doi.org/10.1007/s11606-007-0399-6

29. Esposito K, Marfella R, Ciotola M, Di Palo C, Giugliano F, Giugliano G, et al. Effect of a mediterranean-style diet on endothelial dysfunction and markers of vascular inflammation in the metabolic syndrome a randomized trial. JAMA. 2004;292:1440-6. https://doi.org/10.1001/jama.292.12.1440

30. Esposito K, Ciotola M, Carleo D, Schisano B, Saccomanno F, Sasso FC, et al. Effect of rosiglitazone on endothelial function and inflammatory markers in patients with the metabolic syndrome. Diabetes Care. 2006;29:1071-6. https://doi.org/10.2337/dc05-2174

31. Geluk CA, Asselbergs FW, Hillege HL, Bakker SJ, de Jong PE, Zijlstra F. Impact of statins in microalbuminuric subjects with the metabolic syndrome: A substudy of the PREVEND intervention trial. Eur Heart J. 2005;26:1314-20. https://doi.org/10.1093/eurheartj/ehi253

32. Johnson JL, Slentz CA, Houmard JA, Samsa GP, Duscha BD, Aiken LB, et al. Exercise training amount and intensity effects on metabolic syndrome (from studies of a targeted risk reduction intervention through defined exercise). Am J Cardiol. 2007;100:1759-66. https://doi.org/10.1016/j.amjcard.2007.07.027

33. Orchard TJ, Temprosa M, Goldberg R, Haffner S, Ratner R, Marcovina S, et al. The effect of metformin and intensive lifestyle intervention on the metabolic syndrome: The Diabetes Prevention Program randomized trial. Ann Intern Med. 2005;142:611-9. https://doi.org/10.7326/0003-4819-142-8-200504190-00009

34. Phelan S, Wadden TA, Berkowitz RI, Sarwer DB, Womble LG, Cato RK, et al. Impact of weight loss on the metabolic syndrome. Int J Obes. 2007;31:1442-8. https://doi.org/10.1038/sj.ijo.0803606

35. Ramachandran A, Snehalatha C, Satyavani K, Sivasankari S, Vijay V. Metabolic syndrome does not increase the risk of conversion of impaired glucose tolerance to diabetes in Asian Indians: Result of Indian diabetes prevention programme. Diabetes Res Clin Pract. 2007;76:215-8. https://doi.org/10.1016/j.diabres.2006.08.009 
36. Stewart KJ, Bacher AC, Turner K, Lim JG, Hees PS, Shapiro EP, et al. Exercise and risk factors associated with metabolic syndrome in older adults. Am J Prev Med. 2005;28:9-18. https://doi.org/10.1016/j.amepre.2004.09.006

37. van Gaal LF, Rissanen AM, Scheen AJ, Ziegler O, Rössner S; RIO-Europe Study Group. Effects of the cannabinoid-1 receptor blocker rimonabant on weight reduction and cardiovascular risk factors in overweight patients: 1-year experience from the RIO-Europe study. Lancet. 2005;365:1389-97. https://doi.org/10.1016/S0140-6736(05)66374-X

38. Villareal DT, Miller BV, Banks M, Fontana L, Sinacore DR, Klein S. Effect of lifestyle intervention on metabolic coronary heart disease risk factors in obese older adults. Am J Clin Nutr. 2006;84:1317-23. https://doi.org/10.1093/ajcn/84.6.1317 